\title{
Integrated State Space Reduction for Model Checking Executable Object-Oriented Software System Designs
}

\author{
Fei Xie and James C. Browne \\ Dept. of Computer Sciences \\ Univ. of Texas at Austin, Austin, TX 78712, USA \\ \{feixie, browne\}@cs.utexas.edu Fax: +1 (512) 471-8885
}

\begin{abstract}
This paper presents a general framework for integrated state space reduction in model checking executable object-oriented software system designs. The framework structures the application of state space reduction algorithms into three phases with different algorithms applied in each phase. The interactions between these algorithms are explored to maximize the aggregate effect of state space reduction. Automation support for the framework has been proposed and partially implemented. The framework is presented for system designs modeled in xUML [1] 2], an executable dialect of UML, but can also be used to structure integrated state space reduction for other representations. To further improve the applicability of the framework, domain-specific design patterns can be explored to instantiate the framework for different application domains. An instantiation of the framework for distributed transaction systems is defined and its partial implementation has been applied to the design model of an online ticket sale system. The dimension of software system designs that are model checkable is found to be greatly extended.
\end{abstract}

\section{Introduction}

Executable object-oriented modeling languages such as xUML [1] 2], an executable dialect of UML, are widely applied in industry to model software system designs. Model checking 3] [4 can potentially enhance the reliability and robustness of executable object-oriented software system designs. However, model checking software system designs of arbitrary size is intractable due to the wellknown state space explosion problem. Therefore, state space reduction algorithms have to be applied to reduce the model checking complexity.

Executable object-oriented software system designs are ideal candidates for model checking due to their complete execution semantics and natural incorporation of state models. Furthermore, their major features potentially enable effective state space reductions, for instance, compositional structures may lead to effective decompositions, inheritance relationships may facilitate abstractions, and multiple instances of a class may simplify the identification of symmetries.

This paper defines and describes a general framework for integrated state space reduction in model checking executable object-oriented software system 
designs. The framework assumes that the executable system designs can be translated into model checkable languages and is discussed using system designs modeled in xUML. An earlier paper [5] has been focused on model checking an xUML model by translating the model into the S/R [6] automaton language. Under the framework, state space reduction algorithms are applied in an integrated way to xUML models before and during the translation and to the resulting $\mathrm{S} / \mathrm{R}$ models. Interactions between these algorithms are explored to maximize the aggregate effect of state space reduction.

Many software system designs are constructed following domain-specific design patterns that provide information about structures and behaviors of these systems. Reduction algorithms such as decomposition, abstraction, and symmetry reduction, whose effectiveness depends on structures and behaviors of software systems, can be readily formulated on design models due to the fact that execution behaviors of different components are more observable at the design level and due to the existence of domain-specific design patterns. State space reduction algorithms are often applied in combinations. These facts taken together suggest instantiating the general state space reduction framework for different application domains based on domain specific design patterns.

Distributed transaction systems, which are commonly constructed in a design pattern of dispatchers, agents, and servers with customer initiated transactions as observable units of work, are examples of a family of systems for which a structured process for applying state space reduction algorithms at the design model level can be formulated. This paper illustrates the general framework with its instantiation for distributed transaction systems, a systematic process for reducing model checking a property on the design model of a transaction system to discharging a well-defined set of less complex model checking problems. The process represents a transaction as message sequences, associates the property to be checked with a transaction, partitions the model into sub-models, and decomposes the property into sub-properties and assumptions defined over these sub-models. The process is evaluated by its application in model checking an online ticket sale system. The dimension of transaction systems that can be model checked is materially extended by the systematic process.

There has been extensive research on state space reduction algorithms for either hardware systems or software systems, which is surveyed in [4]. Our work, instead of focusing on particular state space reduction algorithms, explores the integrated application of reduction algorithms in the context of the general framework and investigates how domain specific design patterns can help adapt the general framework to different application domains to achieve more automatic and effective state space reduction. Our work is distinguished from the integrated state space reduction for hardware systems [7] 8] by focusing on software systems and incorporating both reduction algorithms effective for asynchronous semantics and those effective for synchronous semantics.

Section 2 defines the general framework, informally describes the state space reduction algorithms currently applied in the context of the framework, and gives some guidelines for when to apply each state space reduction algorithm and for the application order of these algorithms. Section 3 sketches the partially implemented automation support for the general framework. Section 4 
defines, describes, and illustrates the instantiation of the general framework on distributed transaction systems. Section 5 evaluates the instantiation with results from model checking an online ticket sale system. Section 6 gives the conclusion.

\section{Integrated State Space Reduction}

In this section, a structured framework for integrated application of state space reduction algorithms to executable object-oriented software system designs is defined. The framework is presented for system designs modeled in xUML, but can be used to structure integrated state space reduction for other representations. The state space reduction algorithms being applied in the context of this framework are described and interactions between these algorithms are discussed.

\subsection{General Framework}

The model checking process for an xUML model, previously reported in 5], is a sequential application of the following two procedures on the xUML model:

- xUML-to-S/R translation that translates the $\mathrm{xUML}$ model and an $\mathrm{xUML}$ level query to be checked on the model to an $\mathrm{S} / \mathrm{R}$ model and an $\mathrm{S} / \mathrm{R}$ query;

- S/R level model checking that checks the $\mathrm{S} / \mathrm{R}$ query on the $\mathrm{S} / \mathrm{R}$ model by invoking the COSPAN model checker.

The process, referred to as the basic model checking process in Figure 1, works effectively on xUML models with small numbers of class instances, but cannot scale due to the state space explosion problem. On the other hand, for well-structured xUML models, there are system structure and property specific reduction algorithms at the xUML model level, which cannot be recognized by the xUML-to-S/R translator and the COSPAN model checker, but which can effect major state space reduction on the resulting $\mathrm{S} / \mathrm{R}$ model that is to be model checked. Therefore, the general framework prefaces the basic model checking process with a user-driven state reduction procedure.

The general framework establishes a three-level hierarchy for integrated state space reduction, as shown in Figure1 Different reduction algorithms are invoked on different levels of the hierarchy and applied to models of different forms:

- In the user-driven state space reduction procedure, user-driven reduction algorithms such as decomposition, abstraction, and symmetry reduction are applied to reduce a complex model checking task $T$, a complex query on a complex xUML model, into a set of subtasks. Each subtask checks a subquery of the original query on a sub-model of the original model. A sub-model is either a component or an abstraction of the original model. Each subtask is either discharged by invoking the basic model checking process or further reduced. The reductions applied are validated by invoking the basic model checking process or conducting a simple theorem proving.

- In the xUML-to-S/R translation procedure, automatic reduction algorithms, such as static partial order reduction, are applied, which transform an xUML model prior to its translation into $\mathrm{S} / \mathrm{R}$ with respect to a given $\mathrm{xUML}$ query and construct an equivalent model that has a smaller state space. 


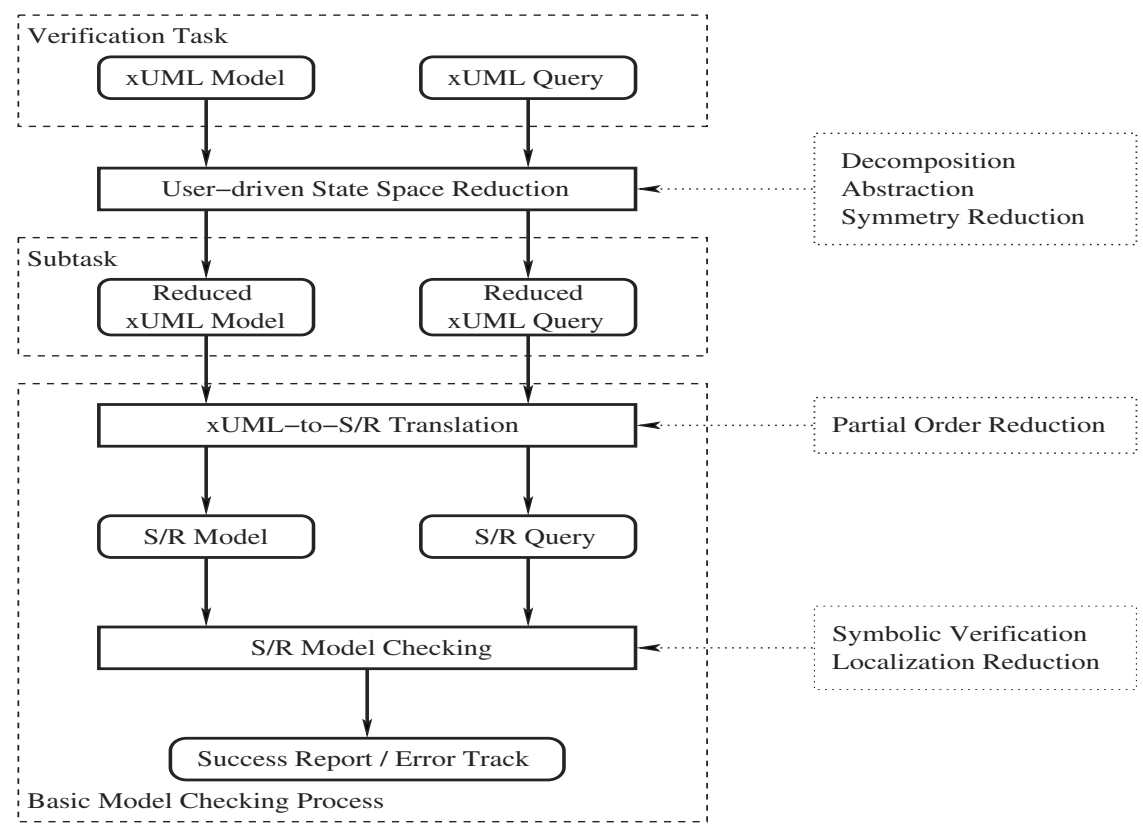

Fig. 1. Reduction Hierarchy of General Framework

- In the S/R level model checking procedure, automatic reduction algorithms implemented by COSPAN, such as symbolic model checking and localization reduction, are applied. These algorithms make use of the semantic information of an $\mathrm{S} / \mathrm{R}$ model to reduce the state space to be explored by COSPAN.

Under the general framework, the extended model checking process for xUML models operates recursively and interactively as shown in Figure 2. A model checking task, $T_{0}$, is recursively reduced into subtasks. A reduction conjecture from users is always validated before its resulting model checking subtasks are discharged. The basic model checking process becomes a model checking engine for discharging subtasks. When a reduction conjecture or a subtask is verified to be false, user interaction is requested. Upon user inputs, either a new reduction conjecture is introduced, or the model checking process is aborted.

\subsection{Major State Space Reduction Algorithms}

There are many possible state space reduction algorithms that can be applied to $\mathrm{xUML}$ models under the general framework. Some of them are summarized as they are applied to xUML models.

Decomposition. The compositional hierarchy, the asynchronous message communication semantics, and the interleaving execution semantics of xUML make decomposition a natural state space reduction algorithm for xUML models. 


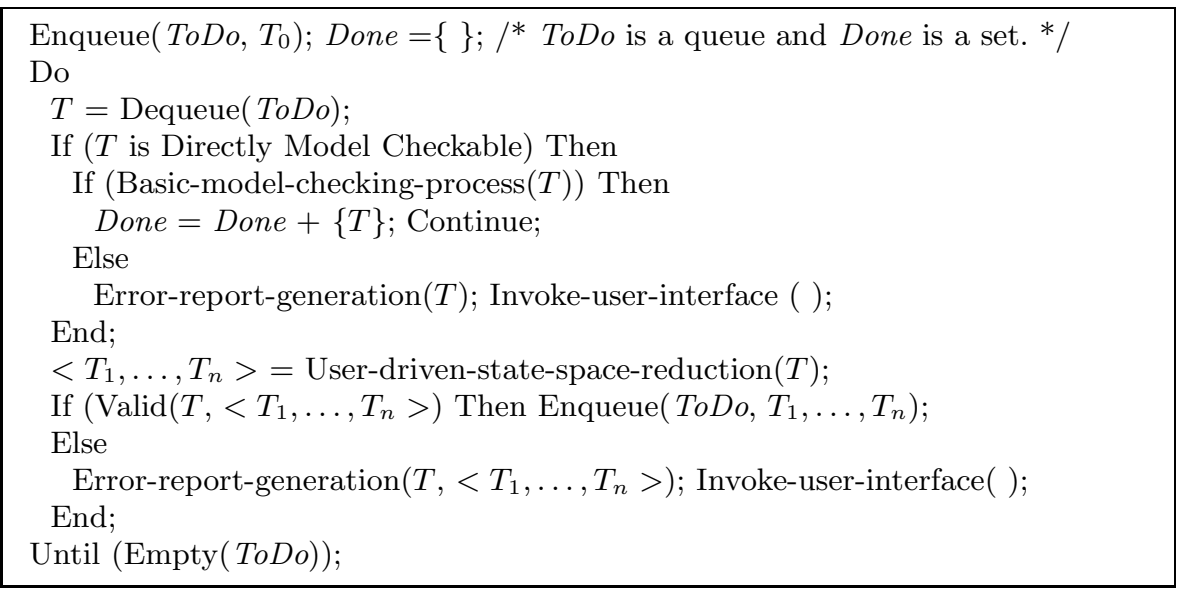

Fig. 2. Recursive and Interactive Model Checking Process Under General Framework

- Query Decomposition. A query on an xUML model can often be broken into a set of sub-queries on the model, its components, or its abstractions so that checking the sub-queries is simpler than checking the original query and verification of the sub-queries guarantees verification of the original query.

- Component-Based Decomposition. To facilitate the query decomposition, the hierarchical structure of an xUML model may be explored to decompose the model into components that have simple and clear interfaces between each other. Dependencies between components are formulated as assumptions of every component on other components. Therefore, a sub-query can be checked on a component under its assumptions, which consumes less memory and time than checking the sub-query on the original model.

- Case Splitting. In many xUML models, concurrent operations may be grouped into units of work, for example, transactions in an e-business system. Commonly there is little interaction between these units of work. If a query on the whole system can be decomposed into sub-queries on units of work and the units of work can be decoupled when these sub-queries are checked, significant state space reduction can often be achieved.

Abstraction. Three abstraction algorithms can be applied:

- State Model Abstraction. If a query is over one or several components of a system, state models in the components not directly involved in the query may be abstracted to reduce the state space to be explored for checking the query. If the abstraction is sound (Executions of the abstract system contain all behaviors of the original system.), then if the query is verified to be true on the abstract system, it will also be true on the original system. The most common form of state model abstraction is the non-deterministic abstraction. For instance, a decision point in a state model may be made non-deterministic 
and a set of state models that are only differentiated by their unique identifiers may be simulated by a state model with a non-deterministic identity. A major advantage of non-deterministic abstraction over other kinds of state model abstraction is that its correctness is automatically guaranteed.

- Data Abstraction. If a mapping can be found between data values of an xUML model and a small set of abstract data values, then an abstract xUML model that simulates the original model can be constructed by extending the mapping to states and transitions. Since the state space of the abstract model is usually smaller, it is often easier to check properties on the abstract model.

- Localization Reduction. Given a model and a property, localization reduction [9], also known as cone of influence reduction [4], eliminates variables in the model that do not influence the variables in the property. The checked property is preserved, but the size of the model to be checked is smaller.

Symmetry Reduction. Symmetry reduction can often reduce the number of queries to be checked on an xUML model or the state space size of the model.

- Symmetric Query Reduction. Given two queries on an xUML model, if a nontrivial mapping can be defined between variables in the model or between values of variables, which maps the model to itself and the two queries to each other, then only one of the two queries need to be checked on the model.

- Quotient Model Reduction. Having symmetry in a model implies the existence of nontrivial permutation groups that preserve both the state labeling and the transition relation. The quotient model induced by this relation is often smaller than the original model. Moreover it is bisimulation equivalent to the original model. Therefore, all queries on the original model can be instead checked on the quotient model.

Partial Order Reduction. Partial order reduction takes advantages of the fact that, in many cases, when components of a system are not tightly coupled, different execution orders of actions or transitions of different components may result in the same global state. Then, under some conditions [10] [11] [12], in particular, when the interim global states are not relevant to the query being checked, model checkers only need to explore one of the possible execution orders. This may radically reduce model checking complexity.

Asynchronous interleaving semantics of xUML suggest application of static partial order reduction [13] to an xUML model prior to its translation into S/R, which transforms the xUML model by restricting its transition structure with respect to a query to be checked. This enables integrated application of partial order reduction while applying symbolic model checking to the $\mathrm{S} / \mathrm{R}$ model.

Symbolic Model Checking. Symbolic model checking represents the state transition structure of an xUML model with binary decision diagrams, which enables manipulation of entire sets of states and transitions instead of individual states and transitions. This heuristic is fully automatic and has shown encouraging reduction promise on some xUML models. (to be elaborated in Section 5). 


\subsection{Interactions between Reduction Algorithms}

Under the general framework, state space reduction algorithms are applied to xUML models in an integrated way. To maximize the aggregate effect of state space reduction, the selection of reduction algorithms and the application order of the selected reduction algorithms need to be carefully considered.

Selection of Reduction Algorithms. The structure of an xUML model and the knowledge of its execution behavior can help select the reduction algorithms to be applied to the model:

a. Symmetry reduction is often selected if there exist many instances of the same class;

b. Partial order reduction is often selected if there is intensive execution interleaving;

c. Symbolic model checking is often selected if there is much randomness.

d. Localization reduction is always applied to $\mathrm{S} / \mathrm{R}$ models.

xUML models from different application domains, different xUML models from the same application domain, or different queries on the same xUML model may lead to different selections of reduction algorithms. Therefore, domain, model, and query specific knowledge also need to be involved in the algorithm selection process besides the selection guidelines provided.

Application Order of Reduction Algorithms. To maximize the state space reduction effect, it is always attempted to apply each reduction algorithm to the minimum models with which the algorithm has to deal. Therefore, the framework hard-codes some application ordering relations between reduction algorithms:

- Algorithms in the user-driven reduction procedure are always applied prior to algorithms in the $\mathrm{xUML}-\mathrm{to}-\mathrm{S} / \mathrm{R}$ translation procedure.

- Algorithms in the xUML-to-S/R translation procedure are always applied prior to algorithms in the $\mathrm{S} / \mathrm{R}$ level model checking procedure.

- In the $\mathrm{S} / \mathrm{R}$ level model checking procedure, localization reduction is always applied prior to symbolic model checking.

There is no ordering relation defined between reduction algorithms applied in the user-driven reduction procedure because the ordering relations between these algorithms are also domain, model, and query specific.

\subsection{Instantiations of General Framework for Application Domains}

The framework defines a general process for structuring integrated state space reductions, but requires certain amount of user interaction. System designs from the same application domains commonly follow a set of domain-specific design patterns and require satisfaction of queries in similar formats. Therefore, domainspecific design patterns and query patterns can often be explored to establish 
an instantiation of the general framework for a given domain. The instantiation should provide additional guidelines for selecting reduction algorithms and additional relations for ordering these reduction algorithms. With these extra efforts, the instantiation may significantly reduce the user interaction required and make the integrated state space reduction for the given domain more automatic and effective. In Section 4, we demonstrate how the general framework is instantiated by instantiating it for distributed transaction systems.

\section{Automation of Integrated State Space Reduction}

Automation support, which is crucial to the wide application of the general framework, is provided through selecting an appropriate model checker, extending the xUML-to-S/R translator, and introducing a reduction manager.

\subsection{Selection of Model Checker}

COSPAN, which has synchronous and parallel semantics, is selected as the model checking engine for xUML models because it supports both symbolic model checking, which is not readily supported by effective model checkers with asynchronous interleaving semantics, and localization reduction. Localization reduction is always applied to any $\mathrm{S} / \mathrm{R}$ model while symbolic model checking can be switched on or off by setting an option of COSPAN.

\subsection{Extension to xUML-to-S/R Translator}

The $\mathrm{xUML}$-to-S/R translator was extended by incorporating the optimization module of SDLCheck [14 that implements static partial order reduction and other software-specific model checking optimizations. These optimizations transforms the $\mathrm{xUML}$ model with respect to the $\mathrm{xUML}$ query before the translation into $\mathrm{S} / \mathrm{R}$ and can be switched on or off without affecting the translation.

\subsection{Reduction Manager}

A reduction manager has been designed and is under development, which coordinates the recursive model checking process in Figure 2 If the current subtask is not directly model checkable, the manager invokes a user interface to input:

- Selected reduction algorithms and their application order;

- Sub-queries of a complex xUML query;

- Boundaries and environment assumptions of a system component;

- Correspondence between sub-queries and components (or units of work);

- Class instances involved in a unit of work;

- Abstract state models and their corresponding concrete state models;

- Abstract data types and their mapping relations to concrete data types;

- Symmetries between class instances (or queries). 
The inputs form a reduction conjecture. The manager applies the selected userdriven reduction algorithms in the user-defined order and generates subtasks. The manager then validates the reduction conjecture by invoking either the basic model checking process or a theorem prover. If the reduction conjecture is not valid, an error handling user interface is invoked to report the error and request a new reduction conjecture or termination of the model checking process.

If the current subtask is model checkable, the manager invokes the basic model checking process to discharge the task. Several tasks can be discharged simultaneously if there is no dependency between them. If a subtask is checked to be false, the manager rolls the whole model checking process back to the reduction that generates the false task

\section{Framework Instantiation on Transaction Systems}

Transaction systems such as banking systems and online sale systems play more and more important roles in the electronic infrastructure of our society. These systems are complex and require high reliability. Their designs follow similar patterns. Therefore, it is worthwhile to instantiate the integrated state space reduction framework for model checking xUML models of transaction systems.

\subsection{Common Patterns of Transaction Systems}

A transaction system executes transactions concurrently. A transaction consists of sequences of interactions between system components. Transactions may be of different types and transactions of the same type are often symmetric. The correctness of the system can be established by determining the correctness of each transaction it performs and the correctness of interactions between transactions.

Definition 1. The model, $M$, of a transaction system, $S$, is the $x U M L$ model of $S$, which consists of a set of interacting class instances. A model, $M^{\prime}$, is a sub-model of $M$ if $M^{\prime}$ consists of a subset of class instances of $M$.

Definition 2. A transaction type, $T$, of $M$ is a message sequence template, which consists of sequences of message types defined in $M$. An instance of $T$ is a transaction executed by $M$, whose message sequences follow T. A type, $T^{\prime}$, is a sub-type of $T$ if each sequence in $T^{\prime}$ is a sub-sequence of a sequence in $T$.

Definition 3. A transaction property, $P$, is a temporal logic predicate over all instances of a transaction type, $T$, or over an instance of $T$.

Definition 4. A model checking task is a tuple, $\langle M, T, P, A\rangle$, where $M$ is a model, $T$ is a transaction type defined on $M, P$ is a transaction property defined on $T$, and $A$ is the set of assumed temporal properties defined on the environment of $M$. The environment of $M$ is the aggregation of all inputs to $M$. A model checking task, $<M^{\prime}, T^{\prime}, P^{\prime}, A^{\prime}>$, is a subtask of $\left.<M, T, P, A\right\rangle$ if $M^{\prime}$ is a sub-model of $M, T^{\prime}$ is a sub-type of $T$, and $P^{\prime}$ is a temporal predicate that 
is defined on $M^{\prime}$ and derived from $P$ through reductions such as decompositions, and $A^{\prime}$ is the union of $A$ and a set of assumed properties on $M-M^{\prime}$. Each assumed property in $A$ or $A^{\prime}$ is a tuple of a temporal predicate and a model (or the environment) on which the predicate is defined.

Definition 5. A model checking task, $\langle M, T, P, A\rangle$, is directly model checkable if it can be discharged by the basic model checking process using a reasonable amount of time and memory.

\subsection{Domain Specific Reduction Algorithm}

The domain specific reduction algorithm for checking a task, $<\hat{M}, \hat{T}, \hat{P}, \hat{A}>$, on a transaction system is given in Figure 3 For simplicity, only the reduction aspect of the algorithm is covered in Figure 3 The algorithm constructs the reduction tree for $<\hat{M}, \hat{T}, \hat{P}, \hat{A}>$ on-the-fly. The root of the tree is $<\hat{M}, \hat{T}, \hat{P}, \hat{A}>$. Each non-root node in the tree is a subtask of its parent. The tree is expanded in a breadth first fashion. Every execution of the do loop either discharges a task at a leaf of the tree or expands the tree by reducing the task into its subtasks through symmetry reduction, decomposition, or case splitting. The expansion stops when all subtasks at the leaves of the tree are directly model checkable.

\subsection{Case Study: An Online Ticket Sale System}

The xUML model of an online ticket sale system [15], $M_{0}$, is employed to illustrate the domain specific reduction algorithm for transaction systems. There are four classes in the system: Customer, Dispatcher, Agent, and Ticket Server. Both the Dispatcher class and the Ticket Server class have only one instance. The Agent class and the Customer class may have an arbitrary number of instances. The system processes ticketing transactions of the type, $T_{0}$, concurrently for many customers. The message sequence diagram of $T_{0}$ is shown in Figure $4 . T_{0}$ has four branching points where the decisions made affect the message sequences:

1. Upon processing a request message from a customer, the dispatcher assigns an idle agent to the customer if there is an idle agent; Otherwise, the dispatcher replies to the customer with a TryLater message;

2. Upon processing a Hold message from an agent, the ticket server replies to the agent with: A Held message if the number of tickets available is greater than the requested number; A Later message if the sum of tickets available or being held is greater than the requested number; An Out message otherwise;

3. Upon receiving a TicketHeld message from an agent, the customer may or may not reply to the agent with its payment;

4. If the valid payment from the customer is received before the agent times out, the agent sends a Ticket message to the customer and a Buy message to the ticket server; Otherwise, it sends a Release message to the ticket server.

A property which should hold on each transaction of the type, $T_{0}$, is that after a request message from a customer is processed by the dispatcher, eventually the 


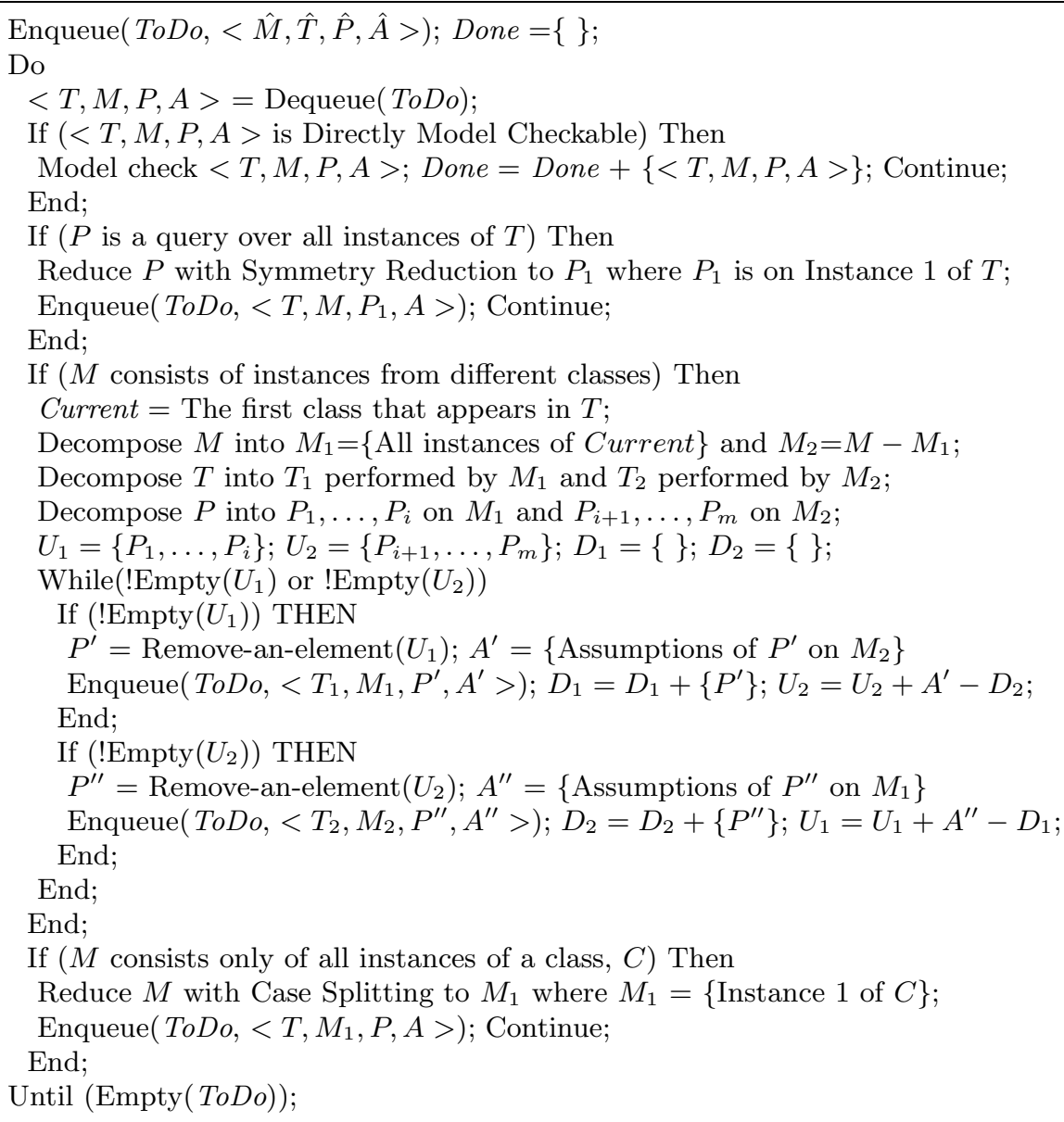

Fig. 3. Domain Specific Reduction Algorithm for Transaction Systems

system will send a TicketHeld message, or a TryLater message, or a SoldOut message back to the customer. The property is formulated as $P_{0}$ in Figure 5 using an xUML level query logic derived from a query logic defined in [7. For simplicity, in Figure 5 some details are left out and $i$ (or $j$ ) is used to index a general instance of the Customer class (or the Agent class, respectively).

Although the structure of the system is simple, the arbitrary number of customers and agents make directly model checking $P_{0}$ infeasible even for the most powerful model checkers. Therefore, the domain specific reduction algorithm is applied to reduce the model checking task, $\left\langle M_{0}, T_{0}, P_{0}, \Phi\right\rangle$. The assumption set is empty since customers are also modeled as class instances in $M_{0}$. The sub-queries involved in the reduction process are defined in Figure 5 The sub transactions and the sub-models involved in the process are shown in Figure 6. The reduction tree generated by the process is shown in Figure 7 Assumptions 


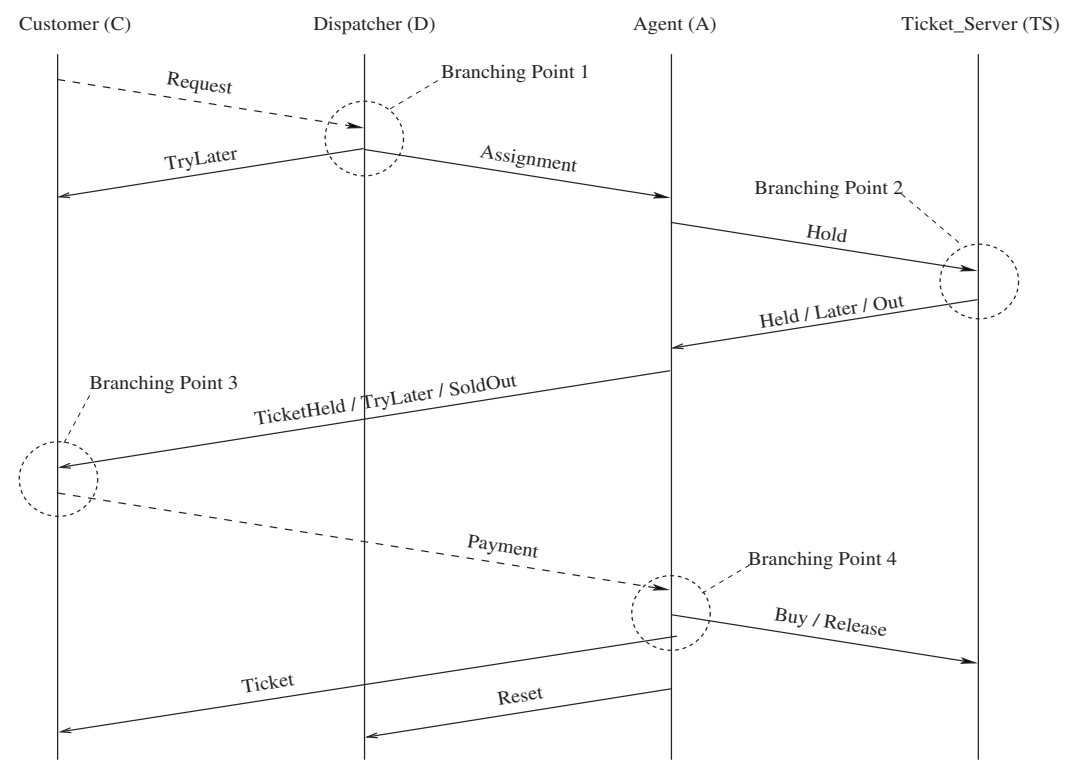

Fig. 4. Message Sequence Diagram of Ticketing Transaction

of a subtask are represented in Figure 7 by dashed arrows which lead to the subtasks that check the assumed properties on the corresponding sub-models. Reductions applied in the process are grouped into six general steps as follows:

Step 1: Symmetry Reduction. $P_{0}$ is a temporal predicate over all transactions of the type $T_{0}$. Since customers are symmetric to each other, checking $P_{0}$ on $M_{0}$ is reduced to checking $P_{1}$ on $M_{0}$ where $P_{1}$ is a predicate only over the transaction that involves Customer 1.

Step 2: Decomposition. $T_{0}$ is decomposed into three sub transaction types, $T_{11}, T_{12}$ and $T_{13}$. Accordingly, $M_{0}$ is decomposed into three sub-models, $M_{11}$, $M_{12}$, and $M_{13}$. Transactions of the types, $T_{11}, T_{12}$ or $T_{13}$, are conducted by $M_{11}$, $M_{12}$, or $M_{13}$ respectively. $P_{1}$ is decomposed into three sub-queries: $P_{21}, P_{22}$, and $P_{23} . P_{21}$ is directly model checkable on $M_{12}$ without any assumption on $M_{11}$ or $M_{13}$. $P_{22}$ is directly model checkable on $M_{12}$ by assuming that $P_{31}, P_{32}$, and $P_{33}$ hold on $M_{13}$.

Step 3: Symmetry Reduction. In $M_{13}$, agents are symmetric. $P_{31}, P_{32}, P_{33}$, and $P_{23}$ have no assumption on $M_{11}$ and $M_{12}$. Therefore, checking $P_{31}, P_{32}, P_{33}$, and $P_{23}$ on $M_{13}$ is reduced to checking $P_{41}, P_{42}, P_{43}$, and $P_{44}$ on $M_{13}$.

Step 4: Decomposition. $T_{13}$ is further decomposed into two sub-types: $T_{21}$ and $T_{22}$. Accordingly, $M_{13}$ is decomposed into two sub-models: $M_{21}$ and $M_{22}$. Transactions of the types $T_{21}$ or $T_{22}$ are conducted by $M_{21}$ or $M_{22}$ respectively. Checking $P_{41}, P_{42}, P_{43}$, and $P_{44}$ on $M_{13}$ is reduced to checking $P_{41}, P_{42}, P_{43}$, and $P_{44}$ on $M_{22}$ by assuming $P_{5}$ holds on $M_{22}$. 


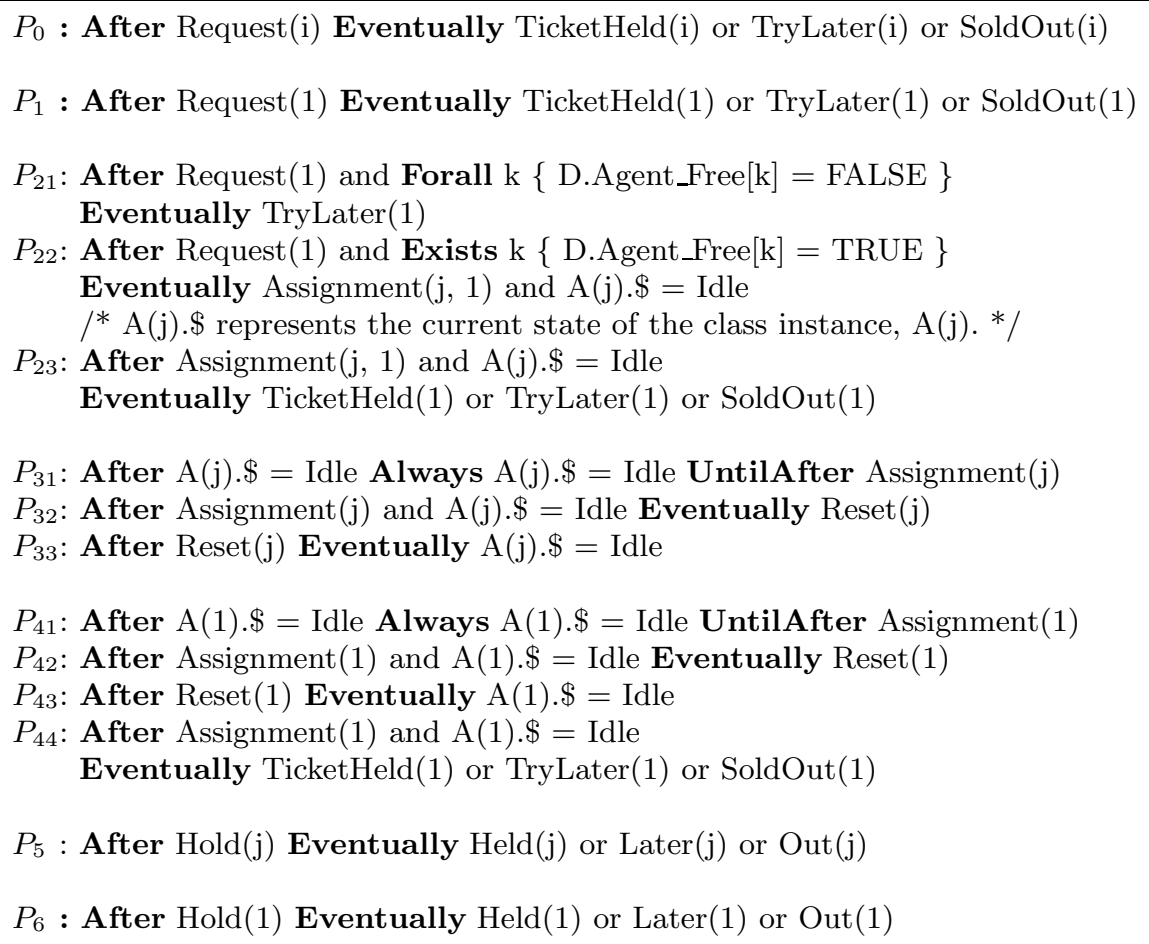

Fig. 5. Original Query and All Intermediate Sub-queries

Step 5: Case Splitting. In $M_{21}$, under the assumption $P_{5}$ on $M_{22}$, transactions of the type $T_{21}$ and performed by agents are independent of each other. Therefore $P_{41}, P_{42}, P_{43}$, and $P_{44}$ is instead checked over $M_{3}$ by assuming $P_{5}$ on $M_{22}$.

Step 6: Symmetry Reduction. In $M_{22}$, transactions of the type, $T_{22}$, are symmetric. Therefore, checking $P_{5}$ on $M_{22}$ is reduced to checking $P_{6}$ on $M_{22}$.

\section{Evaluation of Integrated State Space Reduction}

Under the general framework, reduction algorithms applied in the user-driven reduction procedure recursively break a complex model checking task into subtasks that are directly model checkable while reduction algorithms applied in the other two procedures facilitate directly model checking larger tasks. In this section, experiment results from the model checking study of the online ticket sale system are employed to evaluate the integrated application of these algorithms.

\subsection{Evaluation of User-Driven Reduction Algorithms}

Statistics from model checking Property $P_{0}$ in Figure 5] on the xUML model of the online ticket sale system are employed to demonstrate the effectiveness of 


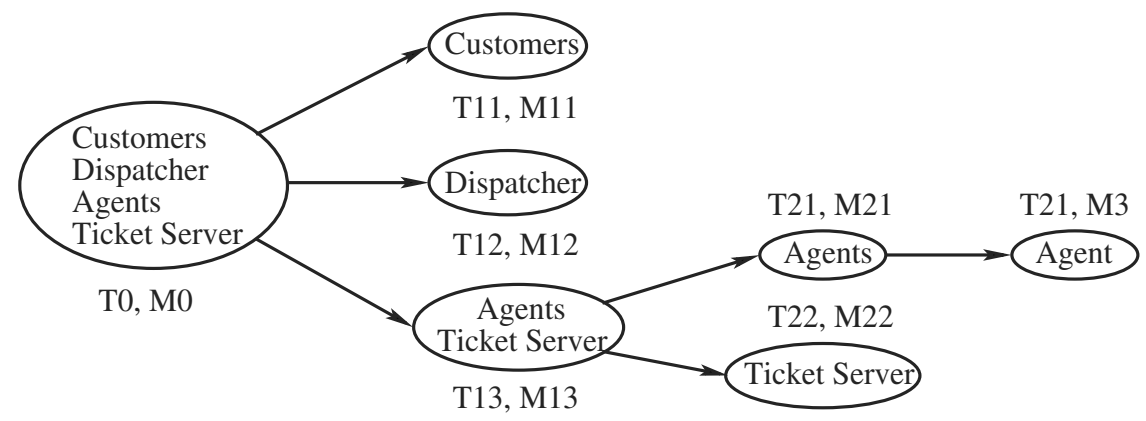

Fig. 6. Decomposition Relations between Sub-models Involved in Reduction

( 1 )

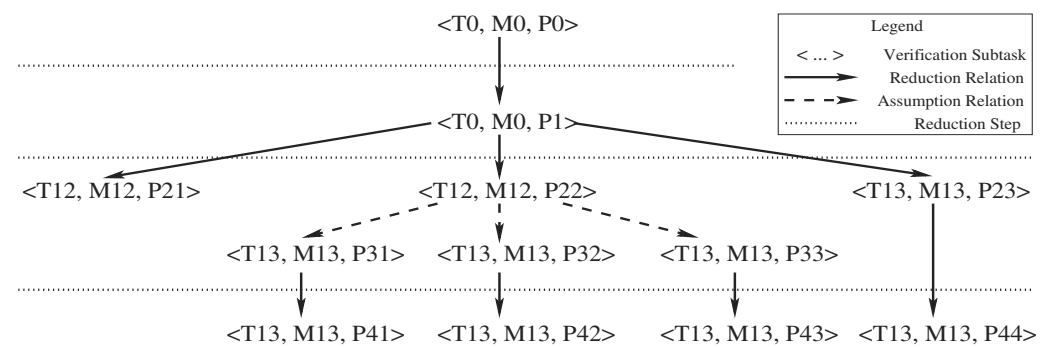

(4)

( 5 )

( 6 )

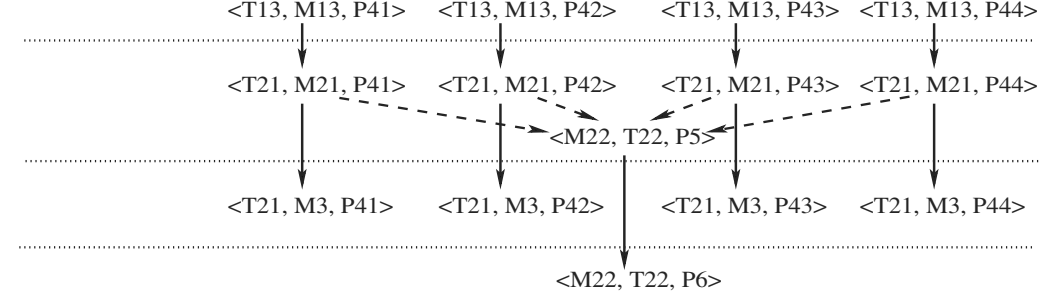

Fig. 7. Reduction Tree for Verifying $P_{0}$ on Online Ticket Sale System

the user-driven reduction algorithms. The memory and time usage for directly model checking $P_{0}$ is compared with the memory and time usage for checking the subtasks generated by applying these reduction algorithms.

Directly model checking $P_{0}$ on the xUML model with two customer instances and two agent instances requires two separate model checking runs, one for each customer instance. With state partial order reduction (SPOR) and symbolic model checking (SMC) applied, each run takes 152.79 megabytes and 16273.7 seconds. The complexity of the xUML model increases rapidly as the number of customers increases. Directly model checking $P_{0}$ on the xUML model with 6 customer instances cannot be fulfilled. Therefore, directly model checking the $\mathrm{xUML}$ model with arbitrary number of customers is not feasible.

The memory and time usage for model checking each subtask from the reduction tree in Figure 7 is shown in Table 1. It can be observed that the memory and time usage for each subtask is substantially lower than that for directly model 
Table 1. Time and Memory Usage of Subtasks in Verifying $P_{0}$

\begin{tabular}{|c|c|c|c|c|c|c|c|}
\hline Criteria & $P_{21}$ & $P_{22}$ & $P_{41}$ & $P_{42}$ & $P_{43}$ & $P_{44}$ & $P_{6}$ \\
\hline Memory & $0.30 \mathrm{M}$ & $0.95 \mathrm{M}$ & $0.28 \mathrm{M}$ & $0.29 \mathrm{M}$ & $0.28 \mathrm{M}$ & $0.29 \mathrm{M}$ & $0.35 \mathrm{M}$ \\
\hline Time & $0.02 \mathrm{~S}$ & $1.81 \mathrm{~S}$ & $0.01 \mathrm{~S}$ & $0.04 \mathrm{~S}$ & $0.01 \mathrm{~S}$ & $0.04 \mathrm{~S}$ & $0.63 \mathrm{~S}$ \\
\hline
\end{tabular}

checking $P_{0}$ on the xUML model with two customers. The model checking result from the reduction process can be scaled up to xUML models with arbitrary number of customer and agent instances by further applying non-deterministic abstraction and symmetry reduction. The complexity of symmetric query reduction is not shown due to an unfinished feature of our reduction system. However, the complexity is theoretically bounded by the complexity of the static structure of an xUML model because the reduction only checks the static structure of the model instead of exploring the full state space of the model.

\subsection{Evaluation of SPOR, SMC, and their Combined Application}

Being able to directly discharge larger model checking tasks reduces user interaction and makes the integrated state space reduction more automatic. Currently, to scale up directly model checkable tasks, SPOR is applied in the xUML-to-S/R translation and SMC is applied in the $\mathrm{S} / \mathrm{R}$ level model checking. To demonstrate

Table 2. Model Checking Memory and Time Usage Comparison

\begin{tabular}{|c|c|c|c|}
\hline SPOR & SMC & Memory Usage & Time Usage \\
\hline Off & Off & $167.072 \mathrm{M}$ & $193748 \mathrm{~S}$ \\
\hline On & Off & $16.0604 \mathrm{M}$ & $10476.5 \mathrm{~S}$ \\
\hline Off & On & $142.746 \mathrm{M}$ & $471.32 \mathrm{~S}$ \\
\hline On & On & $102.527 \mathrm{M}$ & $280.1 \mathrm{~S}$ \\
\hline
\end{tabular}

the reduction ability of SPOR and SMC, Property $P_{21}$ in Figure 5 is directly checked on the whole model under the four possible on/off combinations of SPOR and SMC. The model checking complexities under the four combinations are compared in Table 2. It can be observed that both SPOR and SMC lead to significant reduction on the model checking complexity. SPOR offers a better memory usage while SMC offers a better time usage. Their combined application achieves the best time usage with a medium memory usage.

\section{Conclusion}

This paper defines and describes a general framework for integrated state space reduction in model checking executable object-oriented software system designs. 
The framework is presented for system designs modeled in xUML, but is readily applicable to other representations. Partially implemented automaton support for the framework is discussed. The framework is illustrated by its instantiation for distributed transaction systems and is evaluated by applying the instantiation in model checking an online ticket sale system. The dimension of the software system designs that are model checkable is found to be substantially extended.

\section{Acknowledgment}

We gratefully acknowledge Robert P. Kurshan, Vladimir Levin, Huaiyu Liu, Nancy Macmahon, and Kedar Namjoshi for their generous help.

\section{References}

1. Kennedy Carter: http://www.kc.com/html/xuml.html. Kennedy Carter (2001)

2. Project Tech.: http://www.projtech.com/pubs/xuml.html. Project Tech. (2001)

3. Clarke, E.M., Emerson, E.A.: Design and Synthesis of Synchronization Skeletons Using Branching Time Temporal Logic. Logic of Programs Workshop (1981)

4. Clarke, E.M., Grumberg, O., Peled, D.: Model Checking. The MIT Press (1999)

5. Xie, F., Levin, V., Browne, J.C.: Model Checking for an Executable Subset of UML. Proc. of 16th IEEE International Conf. on Automated Software Engineering (2001)

6. Hardin, R.H., Har'El, Z., Kurshan, R.P.: COSPAN. Proc. of 8th International Conf. on Computer Aided Verification (1996)

7. Cadence: FormalCheck User Guide. Cadence (2001)

8. McMillan, K.L.: A Methodology for Hardware Verification Using Compositional Model Checking. Cadence Technical Report (1999)

9. Kurshan, R.P.: Computer-Aided Verification of Coordinating Processes: The Automata-Theoretic Approach. Princeton University Press (1994)

10. Godefroid, P., Pirottin, D.: Refining Dependencies Improves Partial-Order Verification Methods. 5th International Conf. on Computer Aided Verification (1993)

11. Peled, D.: Combining Partial Order Reductions with On-the-fly Model-Checking. Formal Methods in System Design (1996)

12. Valmari, A.: A Stubborn Attack on State Explosion. Proc. of 2th International Conf. on Computer Aided Verification (1990)

13. Kurshan, R.P., Levin, V., Minea, M., Peled, D., Yenigün, H.: Static Partial Order Reduction. Proc. of 4th International Conf. on Tools and Algorithms for the Construction and Analysis of Systems (1998)

14. Levin, V., Yenigün, H.: SDLCheck: A Model Checking Tool. Proc. of 13th International Conf. on Computer Aided Verification (2001)

15. Wang, W., Hidvegi, Z., Bailey, A.D., Whinston, A.B.: E-Processes Design and Assurance Using Model Checking. IEEE Computer Vol. 33 (2000) 